\title{
Silicosis and methylated arginines/L-arginines: case-control adapted a cross-sectional design
}

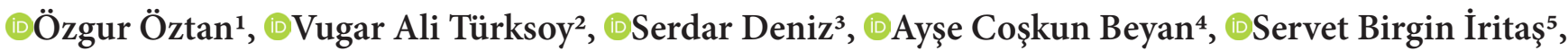 \\ ๑D Müjgan Ercan Karadağ ${ }^{6}$, (D)Engin Tutkun ${ }^{2}$ \\ ${ }^{1}$ HLC Medical Center, Department of Medical Management, Ankara, Turkey \\ ${ }^{2}$ Yozgat Bozok University Faculty of Medicine, Department of Public Health, Yozgat, Turkey \\ ${ }^{3}$ Malatya Turgut Ozal University Faculty of Medicine, Department of Public Health, Malatya, Turkey \\ ${ }^{4}$ Dokuz Eylül University Faculty of Medicine, Department of Occupational Medicine, Turkey \\ ${ }^{5}$ Ankara Institute Forensic Medicine, Ankara, Turkey \\ ${ }^{6}$ Afyon Health Sciences University, Department of Medical Biochemistry, Afyon, Turkey
}

Cite this article as: Öztan Ö, Türksoy VA, Deniz S, et al. Silicosis and methylated arginines/L-arginines: case-control adapted a cross-sectional design. J Health Sci Med 2021; 4(6): 912-918.

\begin{abstract}
Aim: Silicosis has long been recognized as an important occupational lung disease that is included in the group of pneumoconiosis. As already well-known silicosis is a progressive pneumoconiosis characterized by fibrosis in the lungs. Also, chronic exposure to silica may cause chronic obstructive pulmonary disease, emphysema, lung cancer, and pulmonary fibrosis. Asymmetric dimethyl arginine (ADMA), symmetric dimethyl arginine (SDMA), and L-NMMA (NG-mono-methylated-Larginine) are the products of protein arginine methyltransferase (PRMT) enzymes. The aim of this study is to investigate the relationship between silicosis and arginine metabolites in silica exposed and non-exposed workers.

Material and Method: 180 male subjects (90 non-exposed workers (age matched-control) and 90 workers diagnosed with silicosis occupational physician based on radiological and clinical findings and exposure history-(silica-exposed) were included in this study. The serum levels arginine, ADMA, SDMA, and L-NMMA were determined using enzyme-linked immunosorbent assay.

Results: ADMA, SDMA, L-NMMA values were significantly higher in the silica-exposed group compared to the control group. The positive correlations were observed between methylated arginine parameters such as ADMA and SDMA, ADMA and L-NMMA levels $(r=0.43, r=0.60 ; p<0.01)$. The negative correlations were found between SDMA and arginine/ADMA, L-NMMA, and arginine/ADMA, arginine and SDMA/ADMA, arginine/ADMA and SDMA/ADMA levels, respectively $(\mathrm{r}=-0.22 ; \mathrm{r}=-0.22, \mathrm{r}=-0.34, \mathrm{r}=-0.29 ; \mathrm{p}<0.01)$. The strongest positive correlation was found between arginine and arginine/ ADMA ratio, and the strongest negative relationship between ADMA and arginine/ADMA ratio, respectively $(r=0.87 ; \mathrm{r}=$ $-0.48 ; \mathrm{p}<0.01)$.
\end{abstract}

Conclusion: The results could provide additional insight into understanding the disease and the potential for developing biomarkers.

Keywords: Silicosis, arginine, Asymmetric dimethyl arginine, symmetric dimethyl arginine, NG-mono-methylated-L-arginine

\section{INTRODUCTION}

Silicosis has long been recognized as an important occupational lung disease that is included in the group of pneumoconiosis. The risk increases due to the concentration of dust, the percentage of silica in the dust, and the duration of total exposure. The total burden of silica in the body is mainly directly related to the risk of pneumoconiosis. Silicon dioxide has two forms as amorphous and crystalline. The amorphous

form does not seem to cause severe pulmonary disease. Histologically, it can be defined as a diffuse interstitial fibro nodular lung disease. In the post-mortem examinations of silicotic lungs, the tissue is characterized with hilar and parenchymal lymphadenopathies and silicotic nodules, generally located in the upper lobes of the lung. Crystalline silica particles can be seen in a polarized light microscope (1). 
Asymmetric dimethyl arginine (ADMA), symmetric dimethyl arginine (SDMA) and L-NMMA (NG-monomethylated-L-arginine) are the products of protein arginine methyltransferase (PRMT) enzymes (2). As nitric oxide $(\mathrm{NO})$ has reactive oxygen species (ROS) scavenging functions and promotes the activation of endogenous anti-oxidant defense systems, ADMA can contribute ROS imbalance by reducing $\mathrm{NO}$ bioavailability and invoke superoxide production $(3,4)$. The mechanism underlying between silicosis and inflammation is still unknown, but several studies provide many results supporting this link. Many cellular mechanisms can contribute to the inflammation progress in silicosis. The inflammatory cytokines (interleukin (IL)-8, IL-6), arachidonic acid metabolites (leukotrienes, prostaglandins), transcription factors ((nuclear factor kappa-light-chain-enhancer of activated B cells (NFkB) and activator protein-1), and chemokines such as macrophage inflammatory protein (MIP)-2, MIP-1 $\alpha$, MIP- $1 \beta$ and monocyte chemoattractant proteins are involved in the inflammatory process. There are also some experimental studies which have shown to decrease inflammation and production of MIP2 , preventing the subsequent pulmonary fibrosis and silicosis $(1,5)$ ADMA, SDMA and L-NMMA are first extracted in human urine in 1970's (6). Since then, there have been many publications on these metabolites and their relationship with various diseases. Most of them has been highlighted that ADMA contributed in endothelial dysmorphology and can be selected as a screening marker. It has been found to be elevated in the serum in cases of renal insufficiency, cardiovascular diseases and atherosclerosis, renal failure, hyperglycemia and hyperhomocysteinemia $(2,4,6-8)$. The studies evaluating the relationship between arginine metabolites and pulmonary diseases, different hypotheses have been suggested $(11,12)$. However, there is lack of knowledge about the relationship silicosis and arginine metabolites. We aim to identify an expanding role of serum ADMA, SDMA and L-NMMA levels in silica exposed and nonexposed workers.

\section{MATERIAL AND METHOD}

This study was ethically approved by the Noninterventional Clinical Researchs Ethics Committee of the Bozok University Medical Faculty (Date: 17.10.2016, Decision No:17/04). All procedures were carried out in accordance with the ethical rules and the principles of the Declaration of Helsinki.

\section{Study Groups}

180 male subjects (90 non-exposed workers (age matched-control) and 90 workers diagnosed with silicosis occupational physician based on radiological and clinical findings and exposure history-(silica- exposed) were included in this study (13). Serum samples taken as a result of routine examinations were collected from denim bleaching workers diagnosed with silicosis and people in the control group who did not show any symptoms or radiological findings despite working in the same environment. Control group and study group consist of non-smoker or ex-smoker (more than 10 years) workers. In the study, consent forms containing questions about the socio demographic variables, medical history and workplace conditions of both control and exposed groups were filled and strictly adhered to the principles of the Declaration of Helsinki. The workers with chronic diseases (coronary heart disease, hypertension, diabetes, etc.), and acute infections were excluded in the study. The patients were selected among Category 2 patients classified according to ILO pneumoconiosis classification. Oral and written informed consent was granted by all women involved in the study. Serum samples for arginine, ADMA, SDMA, and L-NMMA levels were collected as biological material from the individuals. At the end of the shift week, first morning voiding venous blood were collected into tubes (BD Vacutainer, USA), than centrifuging the samples at $3500 \times \mathrm{g}$ for $10 \mathrm{~min}$ at $4^{\circ} \mathrm{C}$, serum were separated and stored at $-80^{\circ} \mathrm{C}$ further until transfer samples. The samples were transferred in boxes in ice molds to Yozgat Bozok University Science and Technology Application and Research Center (BILTEM) for measuring arginine, ADMA, SDMA and L-NMMA levels.

\section{Laboratory Analysis}

Within the scope of the study, the analysis and preliminary preparation processes of the samples that were thawed in accordance with the commercial kit procedure were performed. The serum arginine, ADMA, SDMA and L-NMMA levels were determined using a commercially available enzyme-linked immunosorbent assay (ELISA) kit, according to the manufacturer's instructions. The reading of the samples placed on the microplates was done with a BMG LABTECH ClarioStar model ELISA device with a wavelength of $450 \mathrm{~nm}$. Control materials used for optimization and for validity of the ELISA methods. Control samples were used for verification. The analysis was done in 5 points according to the calibration curves created against the standard measurements in the ELISA device. Each kit was read at least 10 times and regression analysis was performed by taking the averages.

\section{Statistical Analysis}

Statistical Package for the Social Sciences (IBM SPSS Statistics for Windows, Version 25.0) program was used for the analysis of all parameters. The data were evaluated in terms of compliance with normal distribution using the Kolmogorov-Smirnov test. Descriptive statistics were 
presented with mean, standard deviation, median and minimum-maximum values. Since it was determined that the parameters were not compatible with the normal distribution, the Mann Whitney $U$ test was used to evaluate the status of two independent variables relative to each other. The relationship between variables was determined by Spearman Correlation Analysis. P values of $<0.05$ and $<0.01$ were considered as statistically significant.

\section{RESULTS}

The 90 non-exposed workers and 90 silica-exposed workers included in the study were available for measuring arginine, ADMA, SDMA, and L-NMMA levels. Table 1 shows the differences in methylated arginine between the study groups. Arginine, arginine /ADMA and SDMA/ ADMA values were higher in the silica-exposed group, but this difference was not statistically significant $(p>0.05)$. Also, arginine levels acquired high in exposed groups than control as $84.88 \pm 89.16 \mu \mathrm{mol} / \mathrm{L}$ and $73.79 \pm 44.72$ $\mu \mathrm{mol} / \mathrm{L}(\mathrm{p}>0.05)$. However, ADMA, SDMA, L-NMMA values were significantly higher in the silica-exposed group compared to the control group. The age and body mass index (BMI) were found not different statistically between the groups $(p>0.05)$. The working experience control and silica-exposed groups were observed similar $6.21 \pm 3.31$ years and $5.38 \pm 3.52$ years, respectively ( $p>0.05$ ). On the other hand, median of arginine/ADMA and SDMA/ADMA ratios different groups (control and silica-exposed) were found as 495.94 and $460.49 ; 1.36$ and 1.38 , respectively; but these differences were not statistically significant $(\mathrm{p}>0.05)$.

The Spearman correlations methylated arginine levels showed in Table 2. The positive correlations were observed between methylated arginine parameters such as ADMA and SDMA, ADMA and L-NMMA levels ( $\mathrm{r}=0.43, \mathrm{r}=0.60$; $\mathrm{p}<0.01)$. The negative correlations were found between SDMA and arginine/ADMA, L-NMMA and arginine/ ADMA, arginine and SDMA/ADMA, arginine/ADMA and SDMA/ADMA levels, respectively $(r=-0.22 ; r=$ $-0.22, r=-0.34, r=-0.29, p<0.01)$. The strongest positive correlation was found between arginine and arginine/ ADMA ratio, and the strongest negative relationship between ADMA and arginine/ADMA ratio, respectively

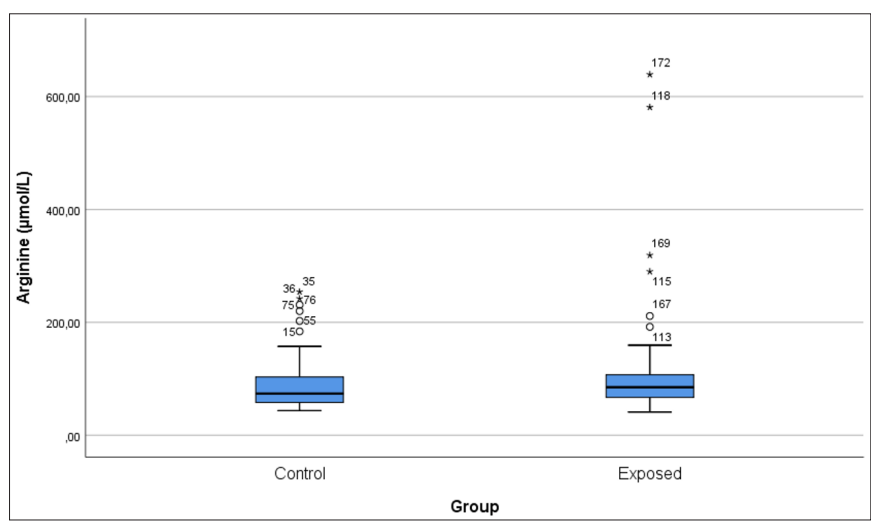

Figure 1. The relationships arginine levels between control and silica-exposed groups. Table 2. The Spearman correlations methylated arginine levels.

\begin{tabular}{|c|c|c|c|c|c|c|}
\hline & Arginine & ADMA & SDMA & L-NMMA & Arginine/ADMA & SDMA/ADMA \\
\hline Arginine & 1 & -0.04 & -0.03 & 0.05 & $.87^{\star \star}$ & $-.34^{* *}$ \\
\hline ADMA & & 1 & $.43^{* *}$ & $.60^{* *}$ & $-.48^{* *}$ & -0.08 \\
\hline SDMA & & & 1 & -0.04 & $-.22^{\star *}$ & -0.02 \\
\hline L-NMMA & & & & 1 & $-.22^{* *}$ & -0.08 \\
\hline Arginine/ADMA & & & & & 1 & $-.29^{* *}$ \\
\hline SDMA/ADMA & & & & & & 1 \\
\hline
\end{tabular}

\begin{tabular}{|c|c|c|c|c|c|c|c|}
\hline & Group" & Mean & Median & Std. Deviation & Minimum & Maximum & $\mathbf{p}$ \\
\hline \multirow{2}{*}{ Arginine $(\mu \mathrm{mol} / \mathrm{L})$} & 0 & 88.98 & 73.79 & 44.72 & 43.50 & 254.10 & \multirow{2}{*}{0.133} \\
\hline & 1 & 105.50 & 84.88 & 89.16 & 41.00 & 639.10 & \\
\hline \multirow{2}{*}{$\mathrm{ADMA}(\mu \mathrm{mol} / \mathrm{L})$} & 0 & 0.16 & 0.16 & 0.03 & 0.11 & 0.28 & \multirow{2}{*}{$0.001^{\star *}$} \\
\hline & 1 & 0.19 & 0.18 & 0.04 & 0.12 & 0.30 & \\
\hline \multirow{2}{*}{$\operatorname{SDMA}(\mu \mathrm{mol} / \mathrm{L})$} & 0 & 0.21 & 0.21 & 0.05 & 0.10 & 0.35 & \multirow{2}{*}{$0.001^{* *}$} \\
\hline & 1 & 0.24 & 0.24 & 0.04 & 0.15 & 0.38 & \\
\hline \multirow{2}{*}{ L-NMMA $(\mu \mathrm{mol} / \mathrm{L})$} & 0 & 0.02 & 0.02 & 0.01 & 0.01 & 0.05 & \multirow{2}{*}{$0.001^{\star *}$} \\
\hline & 1 & 0.03 & 0.03 & 0.01 & 0.02 & 0.06 & \\
\hline \multirow{2}{*}{ Arginine/ADMA ratio } & 0 & 560.74 & 495.94 & 288.06 & 197.09 & 1694.00 & \multirow{2}{*}{0.522} \\
\hline & 1 & 587.65 & 460.49 & 472.15 & 176.10 & 3363.68 & \\
\hline \multirow{2}{*}{ SDMA/ADMA ratio } & 0 & 1.36 & 1.36 & 0.29 & 0.79 & 2.17 & \multirow{2}{*}{0.756} \\
\hline & 1 & 1.37 & 1.38 & 0.34 & 0.61 & 2.21 & \\
\hline
\end{tabular}




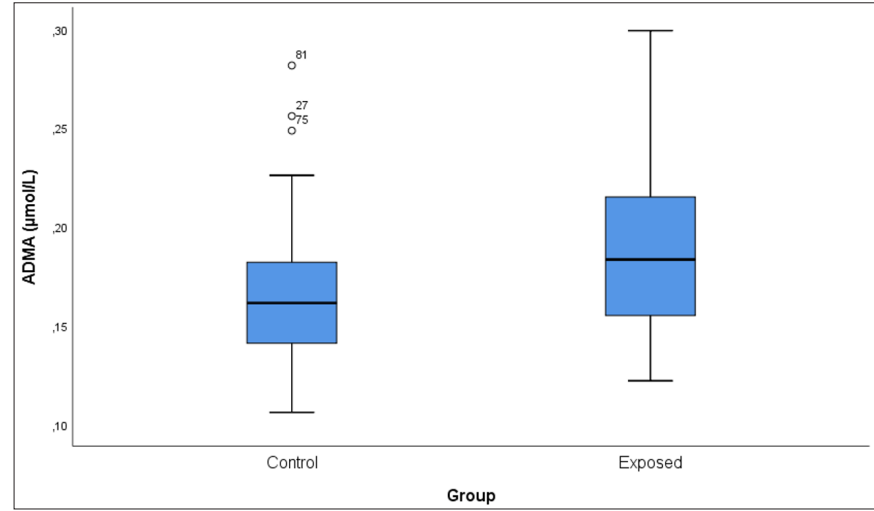

Figure 2. The relationships ADMA levels between control and silicaexposed groups.

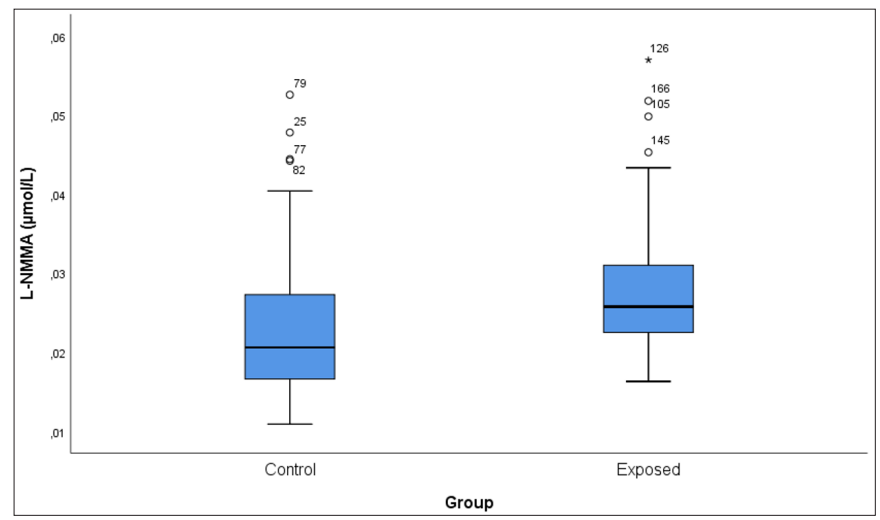

Figure 4. The relationships L-NMMA levels between control and silica-exposed groups.

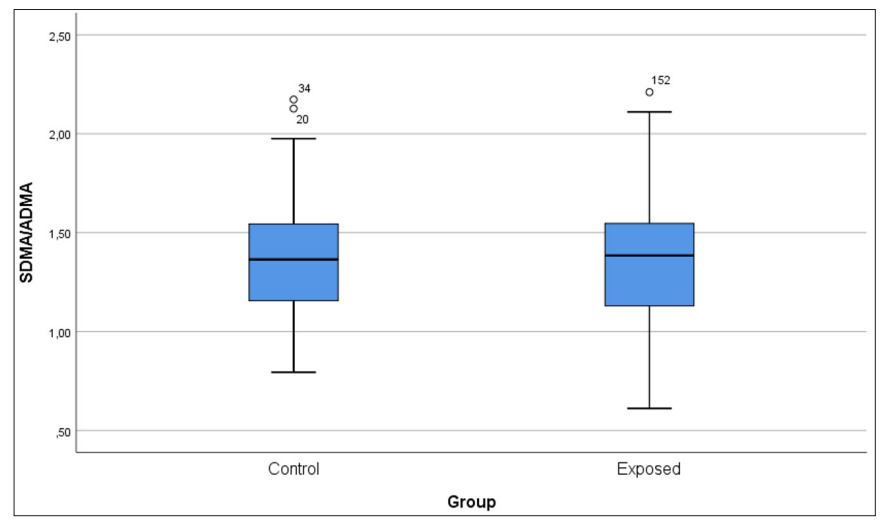

Figure 6. The relationships SDMA/ADMA ratios between control and silica-exposed groups.

$(\mathrm{r}=0.87 ; \mathrm{r}=-0.48 ; \mathrm{p}<0.01)$

\section{DISCUSSION}

The arginine and NO pathway for many diseases and mechanism is well known. ADMA, SDMA, L-NMMA values are significantly higher in the many acute and chronic diseases especially based on hypoxemic mechanism and endothelial dysfunctions $(7,9,14,15)$. Despite, such extensive and comprehensive studies, the role of arginine pathway in the pathogenesis of silicosis has not been studied in detail. In our study, ADMA, SDMA, L-NMMA values were significantly higher in the silica-exposed group compared to the control group.

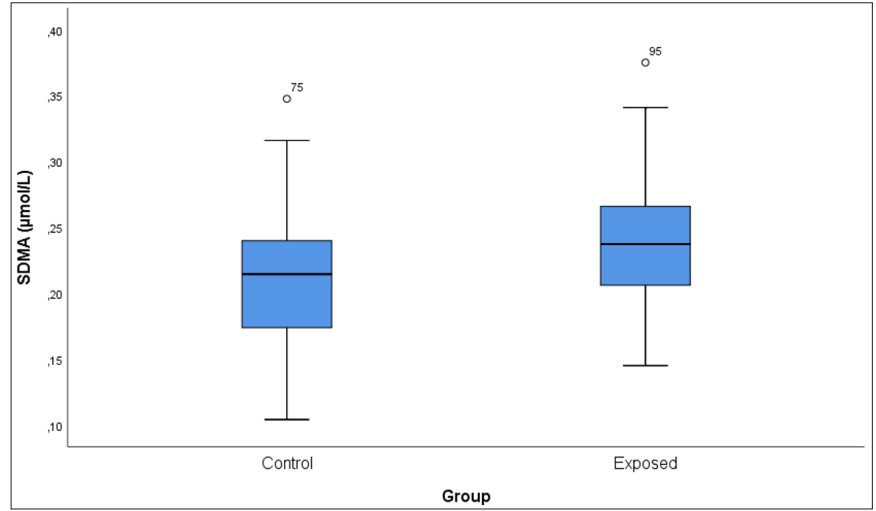

Figure 3. The relationships SDMA levels between control and silicaexposed groups.

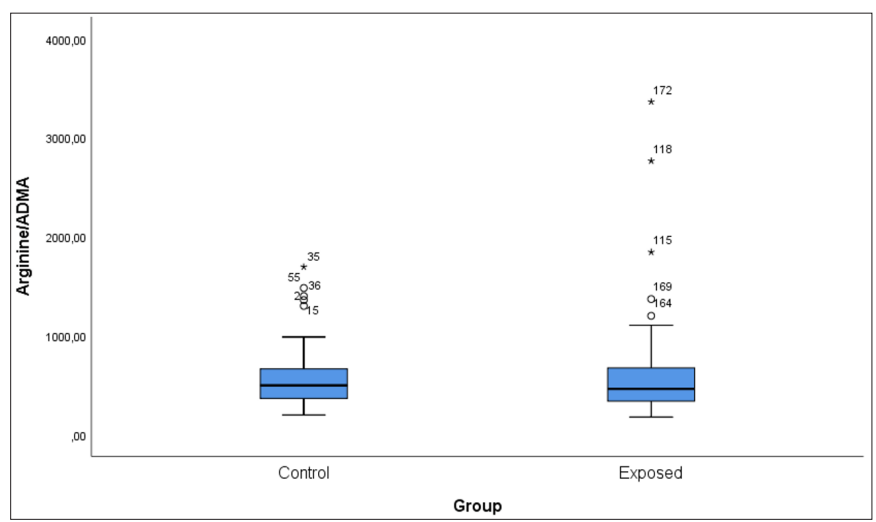

Figure 5. The relationships arginine/ADMA ratios between control and silica-exposed groups.

In contrast to these diseases in which the mechanism of endothelial dysfunction is predominant $(16,17)$, our study provide information regarding the relationship between the toxicity of silica stored in the pulmonary parenchyma and serum ADMA, SDMA and L-NMMA levels. The results not only indicate a correlation between ADMA, SDMA and L-NMMA, and silicosis, but also provide a discussion understanding of the role of the arginine in the silicosis.

The studies evaluating the relationship between arginine metabolites and pulmonary diseases, different hypotheses have been suggested. Airway diseases such as asthma, chronic obstructive pulmonary disease, increased arginase activity in the bronchies may contribute to bronchi obstruction and hyper responsiveness of the airways by reducing of bronchodilatory NO levels and airway remodeling mechanisms. In several animal studies of silicosis, arginase activity and arginase pathway has been also increased in the parenchyma and alveolar macrophages $(16,18-20)$. Wells et al. (11) performed an experimental study by using continuous subcutaneous ADMA infusion to lung fibroblasts and showed altered collagen deposition in the lungs. They state ADMA is major metabolites by inhibiting arginine metabolism in epithelial cells and report that NO is effective on inflammation pathways, as well. Kitowska et al. (21) hypothesize that l-arginine 
metabolism is changed in pulmonary fibrosis by affecting extra cellular matrix synthesis.

As already well known silicosis is a progressive pneumoconiosis characterized by fibrosis in the lungs. Also, chronic exposure to silica may cause chronic obstructive pulmonary disease, emphysema, lung cancer, and pulmonary fibrosis (22). It has complex pathophysiological mechanisms and involves many systematic, cellular and molecular arrangements. Crystalline silica entering the airways, triggers a number of inflammatory processes $(23,24)$. The repeated process of defense circle, the macrophages induces activation of the reactive oxygen radicals and inflammatory reactions, which is linked with pulmonary interstitial fibrosis mechanism $(25,26)$. On the other hands, the role of arginine pathway in the pathogenesis of pneumoconiosis has not been studied in detail. Xue et al. (27) studied distinct metabolic features in the silicosis. They stated sphingolipid and arginine and proline metabolism were the dominant metabolic pathway in silicosis and arginine was positively correlated with the stage of silicosis. Increased levels of some inflammatory markers IL- 8 and IL- 6 , tumor necrosis factor, and IL- $1 \beta$, -were elevated in fibrotic lung tissue of silica-induced lung damage compared with normal lung tissue. (clear form of abbreviations should be stated where they are first used.) In the early pathogenesis of silicosis, the enhancement of arginine metabolism and is closely related to the severity of the fibrosis $(28,29)$ In the end stage, the formation of silicotic micro nodules is the pathological manifestation of the production of collagen bres and pulmonary interstitial fibrosis mediated by amino acid metabolism, which is consistent with the recent studies that arginine pathway and extracellular matrix synthesis were the dominant mechanism in silicosis (27).

ADMA is similar to arginine in terms of molecular structure and it competes with arginine for NOS binding, thus blocking the production of $\mathrm{NO}$ from arginine by NOS directly. Since, ADMA competes with arginine for NOS, the bioavailability of NO depends on the equation between the two, the so-named arginine/ADMA ratio (30). Keller et al. (31) stated that the arginine/ADNA ratio is a more sensitive risk marker in cardiac diseases. In our study arginine/ADMA and SDMA/ADMA ratios were not significantly different exposed versus non exposed groups. Also in correlation analysis we found positive correlation between arginine and arginine/ADMA ratio. Molnar et al. (14) assessed L-arginine, arginine/ADMA ratio in stroke patients. They stated that arginine levels may tend to both increase and decrease and different results can be found in different stages of silicosis.

Arginine, ADMA, SDMA, L-NMMA studies show improvement $(32,33)$. An increasing number of studies have been reported on the use them in the early diagnosis, treatment and follow-up of diseases. Major promising improvement has been made in research on ADMAlowering therapies in cancer, cardiovascular diseases and so on. However, further human studies are needed in order to treat human diseases related to elevated ADMA/ SDMA levels (34-36). Furthermore, the studies that L-arginine may have a predictive role in monitoring silicosis and many other diseases, as well $(27,37)$. Several studies stated that ADMA and oxidative stress biomarkers as a screening biomarker for pulmonary arterial hypertension and silicosis, COPD respectively (38-40). Pacheco et al. (41) stated macrophages polarize to M2 phenotype in response to arginase, and in this case they are involved in the inflammation resolution and tissue repairs. However, different results regarding these studies are also reported (17). Further evaluations with larger study groups are necessary.

\section{Strengths and Limitations}

Some strengths and limitations should be discussed. The case and control group matched in terms of age and working year were included in the study are the strengths of this study, as it reduces confounding factors. On the other hand, despite the absence of environmental silica measurements, the absence of an evaluation of the disease stages of silicosis patients with a relatively small number of cases are main limitations in our study. Finally, given the cross-sectional design, the study has limited power to explain the potential metabolic biomarkers for disease progression and survival, which are clinically significant.

\section{CONCLUSION}

In conclusion, the diagnosis and screening of silicosis basically depend on a history of silica exposure and radiologicalfindings. Recentstudieson pathophysiological mechanisms and markers to understand the nature of silicosis have a great contribution to better understanding silicosis. Our small study showed the relationship between silicosis and arginine pathway. The results could provide additional insight into understanding the disease and potential for developing biomarkers. We believe that the role of ADMA, SDMA and arginine in the diagnosis, screening, treatment and follow-up of silicosis will be better understood with future studies.

\section{ETHICAL DECLARATIONS}

Ethics Committee Approval: This study was ethically approved by the Non-interventional Clinical Researchs Ethics Committee of Bozok University Medical Faculty (Date: 17.10.2016, Decision No:17/04).

Informed Consent: All patients signed the free and informed consent form. 
Referee Evaluation Process: Externally peer-reviewed. Conflict of Interest Statement: The authors have no conflicts of interest to declare.

Financial Disclosure: This study was supported by the Bozok University Scientific Research Foundation (grant No. 6602b-TF/18-227).

Author Contributions: All of the authors declare that they have all participated in the design, execution, and analysis of the paper, and that they have approved the final version.

\section{REFERENCES}

1. Chan JYW, Tsui JCC, Law PTW, et al. Regulation of TLR4 in silicainduced inflammation: An underlying mechanism of silicosis. Int J Med Sci 2018; 15: 986-91.

2. Dowsett L, Higgins E, Alanazi S, Alshuwayer NA, Leiper FC, Leiper J. ADMA: a key player in the relationship between vascular dysfunction and inflammation in atherosclerosis. J Clin Med 2020; 9: 3026.

3. Patel RP, Levonen AL, Crawford JH, Darley-Usmar VM. Mechanisms of the pro- and anti-oxidant actions of nitric oxide in atherosclerosis. Vol. 47, Cardiovascular Research. Oxford Academic; 2000. p. 465-74.

4. Antoniades C, Shirodaria C, Leeson P, et al. Association of plasma asymmetrical dimethylarginine (ADMA) with elevated vascular superoxide production and endothelial nitric oxide synthase uncoupling: Implications for endothelial function in human atherosclerosis. Eur Heart J 2009; 30: 1142-50.

5. Zhao Y, Hao C, Bao L, et al. Silica particles disorganize the polarization of pulmonary macrophages in mice. Ecotoxicol Environ Saf 2020; 193.

6. Oliva-Damaso E, Oliva-Damaso N, Rodriguez-Esparragon F, et al. Asymmetric (ADMA) and symmetric (SDMA) dimethylarginines in chronic kidney disease: A clinical approach. Int J Molecular Sci. MDPI AG 2019; 20: 3668.

7. Koudelka A, Ambrozova G, Klinke A, et al. Nitro-oleic acid prevents hypoxia- and asymmetric dimethylarginine-induced pulmonary endothelial dysfunction. Cardiovasc Drugs Ther 2016; 30: 579-86.

8. Vallance P, Leiper J. Cardiovascular biology of the asymmetric dimethylarginine: dimethylarginine dimethylaminohydrolase pathway. Arterioscler Thromb Vasc Biol 2004; 24: 1023-30.

9. Zewinger S, Kleber ME, Rohrer L, et al. Symmetric dimethylarginine, high-density lipoproteins and cardiovascular disease. Eur Heart J 2017; 38: 1597-607.

10.Szabo Z, Bartha E, Nagy L, Molnar T. Increased symmetric dimethylarginine, but not asymmetric dimethylarginine, concentrations are associated with transient myocardial ischemia and predict outcome. J Int Med Res 2020; 48: 6.

11. Wells SM, Buford MC, Migliaccio CT, Holian A. Elevated asymmetric dimethylarginine alters lung function and induces collagen deposition in mice. Am J Respir Cell Mol Biol 2009; 40: 179-88.

12. Vögeli A, Ottiger M, Meier MA, et al. Asymmetric Dimethylarginine Predicts long-term outcome in patients with acute exacerbation of chronic obstructive pulmonary disease. Lung 2017; 195: 717-27.

13. Fernández Álvarez R, Martínez González C, Quero Martínez A, Blanco Pérez JJ, Carazo Fernández L, Prieto Fernández A. Guidelines for the Diagnosis and Monitoring of Silicosis. Arch Bronconeumol 2015; 51: 86-93.

14. Molnar T, Pusch G, Papp V, et al. The L-arginine pathway in acute ischemic stroke and severe carotid stenosis: Temporal profiles and association with biomarkers and outcome. J Stroke Cerebrovasc Dis 2014; 23: 2206-14.
15. Hosinian M, Qujeq D, Ahmadi Ahangar A. The relation between GABA and L-arginine levels with some stroke risk factors in acute ischemic stroke patients. Int J Mol Cell Med Spring 2016; 5: 100-5.

16. Maarsingh H, Pera T, Meurs H. Arginase and pulmonary diseases. Vol. 378, Naunyn-Schmiedeberg's Archives of Pharmacology 2008. p. 171-84.

17. Parmaksiz E, Inal A, Salepci B, et al. Relationship of asymmetric dimethylarginine levels with disease severity and pulmonary hypertension in chronic obstructive pulmonary disease. Lung India 2018; 35: 199-203.

18. Tadié JM, Henno P, Leroy I, et al. Role of nitric oxide synthase/ arginase balance in bronchial reactivity in patients with chronic obstructive pulmonary disease. Am J Physiol - Lung Cell Mol Physiol 2008; 294: 3.

19. Fajardo I, Svensson L, Bucht A, Pejler G. Increased levels of hypoxiasensitive proteins in allergic airway inflammation. Am J Respir Crit Care Med 2004; 170: 477-84.

20.Böger R, Hannemann J. Dual role of the L-arginine-ADMANO pathway in systemic hypoxic vasodilation and pulmonary hypoxic vasoconstriction. Vol. 10, Pulmonary Circulation. SAGE Publications Ltd; 2020. Apr-Jun; 10: 2.

21. Kitowska K, Zakrzewicz D, Königshoff M, et al. Functional role and species-specific contribution of arginases in pulmonary fibrosis. Am J Physiol Cell Mol Physiol 2008; 294: L34-45.

22.Ding M, Chen F, Shi X, Yucesoy B, Mossman B, Vallyathan V. Diseases caused by silica: Mechanisms of injury and disease development. Vol. 2, International Immunopharmacology. Elsevier; 2002. p. 173-82.

23. Rimal B, Greenberg AK, Rom N. Basic pathogenetic mechanisms in silicosis: Current understanding. Vol. 11, Current Opinion in Pulmonary Medicine. Lippincott Williams and Wilkins; 2005. p. 169-73.

24. Anlar HG, Bacanli M, İitaş S, et al. Effects of Occupational Silica Exposure on OXIDATIVE Stress and Immune System Parameters in Ceramic Workers in TURKEY. J Toxicol Environ Heal - Part A Curr Issues 2017; 80: 688-96.

25. Pollard KM. Silica, silicosis, and autoimmunity. Front Immunol 2016; 7: 97.

26. Liu H, Cheng Y, Yang J, et al. BBC3 in macrophages promoted pulmonary fibrosis development through inducing autophagy during silicosis. Cell Death Dis 2017; 8: 3 .

27. Xue C, Chao-Yang B, Alicated H, et al. Distinct metabolic features in the plasma of patients with silicosis and dust-exposed workers in Chinaa case-control study. BMC Pulmonary Medicine 2021; 21.

28. Misson P, van den Brûle S, Barbarin V, Lison D, Huaux F. Markers of macrophage differentiation in experimental silicosis. J Leukoc Biol 2004; 76: 926-32.

29. Miao R, Ding B, Zhang Y, Xia Q, Li Y, Zhu B. Proteomic profiling change during the early development of silicosis disease. J Thorac Dis 2016; 8: 329-41.

30. Brinkmann SJH, Wörner EA, Buijs N, et al. The arginine/ADMA ratio is related to the prevention of atherosclerotic plaques in hypercholesterolemic rabbits when giving a combined therapy with atorvastatine and arginine. Int J Mol Sci 2015; 16: 12230-42.

31. Notsu Y, Yano S, Shibata H, Nagai A, Nabika T. Plasma arginine/ ADMA ratio as a sensitive risk marker for atherosclerosis: Shimane CoHRE study. Atherosclerosis 2015; 239: 61-6.

32. Clemente GS, van Waarde A, Antunes IF, Dömling A, Elsinga $\mathrm{PH}$. Arginase as a potential biomarker of disease progression: A molecular imaging perspective. Vol. 21, International Journal of Molecular Sciences. MDPI AG; 2020. p. 1-36.

33. Mussai F, Wheat R, Sarrou E, et al. Targeting the arginine metabolic brake enhances immunotherapy for leukaemia. Int J Cancer 2019; 145: 2201-8.

34. Tain YL, Hsu CN. Toxic dimethylarginines: Asymmetric dimethylarginine (ADMA) and symmetric dimethylarginine (SDMA). Toxins (Basel) 2017; 9: 92. 
35. Trocha M, Szuba A, Merwid-Lad A, Sozánski T. Effect of selected drugs on plasma asymmetric dimethylarginine (ADMA) levels. Pharmazie 2010; 65: 562-71.

36. Gao L, Zhang JH, Chen XX, et al. Combination of L-Arginine and L-Norvaline protects against pulmonary fibrosis progression induced by bleomycin in mice. Biomed Pharmacother 2019; 113: 108768 .

37. Böger RH, Maas R, Schulze F, Schwedhelm E. Elevated levels of asymmetric dimethylarginine (ADMA) as a marker for cardiovascular disease and mortality. Clin Chem Lab Med 2005; 43: 1124-9.

38. Thakkar V, Stevens WM, Prior D, et al. N-terminal pro-brain natriuretic peptide in a novel screening algorithm for pulmonary arterial hypertension in systemic sclerosis: A case-control study. Arthritis Res Ther 2012; 14: 3.

39. Nardi J, Nascimento S, Göethel G, et al. Inflammatory and oxidative stress parameters as potential early biomarkers for silicosis. Clinica Chimica Acta 2018; 484: 305-13.

40. Telo S, Kırkıl G, Kuluöztürk M, Balin M, Deveci F. Can ADMA play a role in determining pulmonary hypertension related to chronic obstructive pulmonary disease? Clin Respir J 2018; 12: 1433-8.

41.Lopes-Pacheco M, Bandeira E, Morales MM. Cell-based therapy for silicosis. Stem Cells International 2016; doi. org/10.1155/2016/5091838 